\title{
Damped vibrations method for measuring the rigidity of a system of movable frames of a current collector on the roof of an electric locomotive
}

\author{
Alexander Smerdin ${ }^{1, *}$, Anton Golubkov ${ }^{1}$, Oleg Sidorov ${ }^{1}$, and Anatoly Slatin ${ }^{1}$ \\ ${ }^{1}$ Omsk State Transport University (OSTU), 35, Marx st., 644046, Omsk, Russian Federation
}

\begin{abstract}
The paper describes the features of using the method of damped mechanical vibrations for express diagnostics of current collectors without removing from electric rolling stock roof. The proposed method is based on preliminary determination of the mechanical characteristics of the system of movable frames of a serviceable current collector in the factory. The paper describes a technique that can significantly reduce the complexity of conducting this type of research, increase the speed of measurement, and reduce the influence of the human factor.
\end{abstract}

\section{Introduction}

Global trends in increasing railway transport speeds are also reflected in the "Strategy for the development of railway transport in the Russian Federation until 2030", approved by decree of the Government of the Russian Federation No 878-R of June 17, 2008 [1], according to which, one of the priority areas of development is to increase the efficiency of electric rolling stock. The creation of new equipment for transport systems is included in the List of priority areas for the development of science, technology and machinery of the Russian Federation (approved by the Decree of the President of the Russian Federation of July 07, 2011 [2]), increasing the readiness of the transport industry for import substitution, reducing dependence on supplies from abroad, which is an urgent issue in the current geopolitical and epidemiological situation.

Due to the widespread use of single-lever current collectors on electric rolling stock of both passenger (figure 1b,c,d) and cargo (figure 1a) traffic, the issues of improving their design and operation technology are particularly relevant. An important advantage of current collectors of this type is the reduced metal consumption, their dimensions allow for more rational placement of roof equipment on an electric locomotive, and reducing the number of levers gives certain advantages in terms of aerodynamics.

A distinctive feature of single-lever current collectors is the lack of developed kinematic connections that ensure the stability of the system of movable frames under the action of transverse and longitudinal loads. These types of loads lead to elastic deformations of the lower and upper levers. The resulting elastic forces prevent further growth of displacements.

\footnotetext{
${ }^{*}$ Corresponding author: alexandr.smerdin@omgups.com
} 


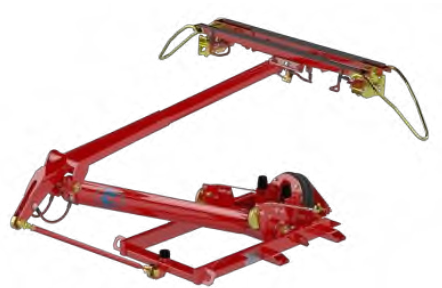

a

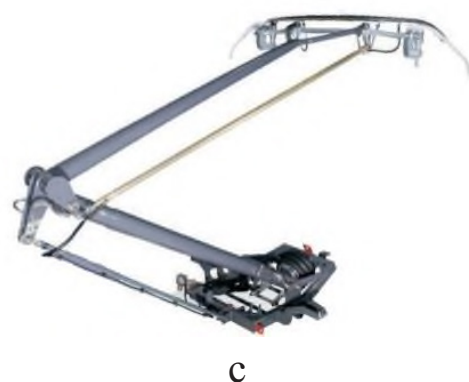

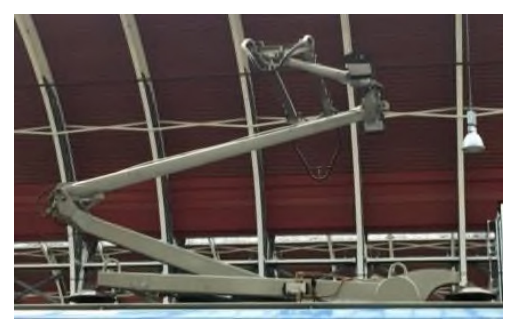

b

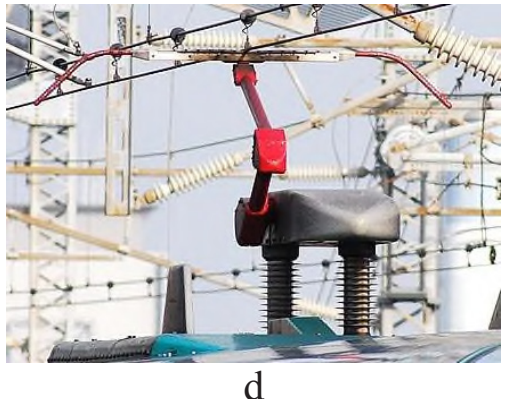

Fig.1. Lever type current collectors designed for operation at various speed ranges: a - up to 160 $\mathrm{km} / \mathrm{h} ; \mathrm{b}$ - up to $200 \mathrm{~km} / \mathrm{h}$; c - up to $300 \mathrm{~km} / \mathrm{h} ; \mathrm{d}$ - over $300 \mathrm{~km} / \mathrm{h}$.

When the transverse rigidity decreases, the current collector of an electric rolling stock under the influence of loads from the contact network is deformed, the slide is shifted relative to the contact wire by an unacceptable distance. As a result of the contact wires coming off the working surface of the current collector, emergency situations occur, which result in interruptions in the movement of trains on the damaged section.

The range of changes in the working height of current collectors in accordance with the international standard GOST 32204-2013 "Current collectors of railway electric rolling stock" is $1500 \mathrm{~mm}$, from 400 to $1900 \mathrm{~mm}$ from the level of the folded position [3]. Spherical joints ensure the movement of the slide in the vertical plane and at the same time transmit loads in the transverse one. Therefore, to control the strength characteristics, it is sufficient to apply a normalized force to the upper node of the movable frame system and measure the deformation caused by this force. The transverse rigidity must be within the standard values over the entire range of changes of the working height (figure 2).

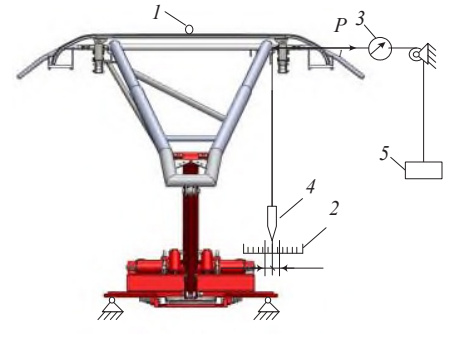

a

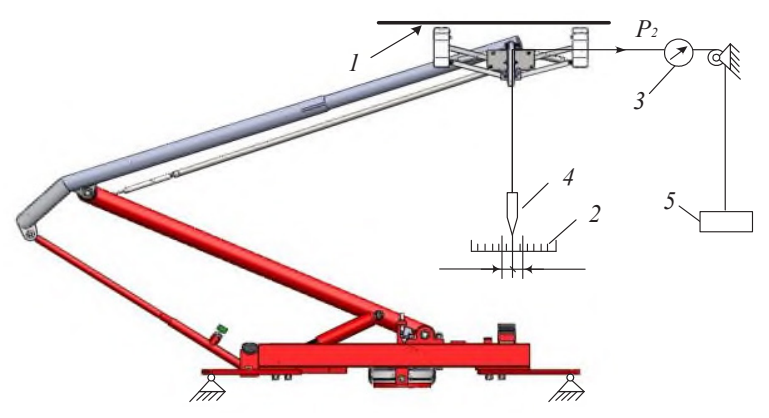

b

Fig. 2. Determination of the transverse (a) and longitudinal (b) rigidity of the current collector on the stand: 1 - contact wire of the beam of the shock-vibration complex; 2 - measuring ruler; 3 dynamometer; 4 - plumb line; 5 - model loads $2 \times 25 \mathrm{~kg}$. 
The traditional method of measurement involves removing the current collector from the electric locomotive and placing it on a specialized stand [4], which is associated with significant labor costs.

\section{Materials and methods}

As part of the study, it is proposed to improve the method for determining the transverse rigidity using vibration diagnostics methods.

This method of rigidity control is based on known regularities of changes in the amplitude-frequency characteristics of the vibration process (the main or resonant frequency of vibrations, the amplitude and logarithmic decrement of vibrations) of natural or forced mechanical transverse or longitudinal vibrations when the strength parameters of the sample change [5-7].

The proposed method does not require removing the current collector from the roof of an electric rolling stock, since the mass of a stationary electric locomotive or an electric train car is very significant compared to the mass of the vibrating parts of the current collector.

Damped vibrations caused by a single shock impact applied to the upper node of the system of movable frames of the current collector must be registered with the maximum available sampling of the signal, and then, by analytical processing of the obtained vibrograms and spectrograms, the desired values of the characteristics of the vibration process must be selected.

Given the assumptions that the cross section of the lower and upper levers of the system of movable frames of the current collector are not changed in length, and viscous friction and flexural rigidity are considered by given to the upper node of the lumped elements [8], the differential equation describing the damped vibrations of the top node of the system of movable frames after a single shock, can be written in the following form:

$$
\begin{gathered}
J_{m} \cdot \ddot{\varphi}=M_{m g}+M_{\text {elas }}+M_{\text {tr }} \\
\ddot{\varphi}=\frac{\left(g \cdot\left(\left(m_{1}+m_{2}\right) \cdot h_{1}+m_{0} \cdot\left(h_{1}+h_{2}\right)\right)-k \cdot\left(h_{1}+h_{2}\right)^{2}\right) \cdot \varphi}{J_{m}} \\
+\frac{b \cdot\left(h_{1}+h_{2}\right) \cdot \dot{\varphi}}{J_{m}}
\end{gathered}
$$

where $J_{m^{-}}$is the moment of inertia of system of movable frames; $\varphi-$ is the generalized coordinate (the angle of deflection of the movable frames system from the vertical); $M_{m g}-$ the moment created by the force of gravity; $M_{\text {elas }}$ - the moment created by the force of elasticity; $M_{t r}$ - the moment produced by the dissipative forces; $\mathrm{b}$ - the coefficient of viscous friction; $h_{1}$ - length of the projection of the lower lever $l_{1}$ on the vertical axis; $h_{2}-$ length of the projection of the upper lever $l_{2}$ on the vertical axis; $k$ - is the transverse rigidity of the system of movable frames.

To increase the speed of testing in a depot or maintenance facility, it is advisable to determine the rigidity of the system of movable frames of the current collectors without dismantling the slide. In this case, it will need to be fixed with clamps relative to the upper node of the system of movable frame.

In the framework of the problem to be solved, the slide is proposed to be represented as a rectangular cross-section beam with a uniformly distributed mass (figure 3 ). 


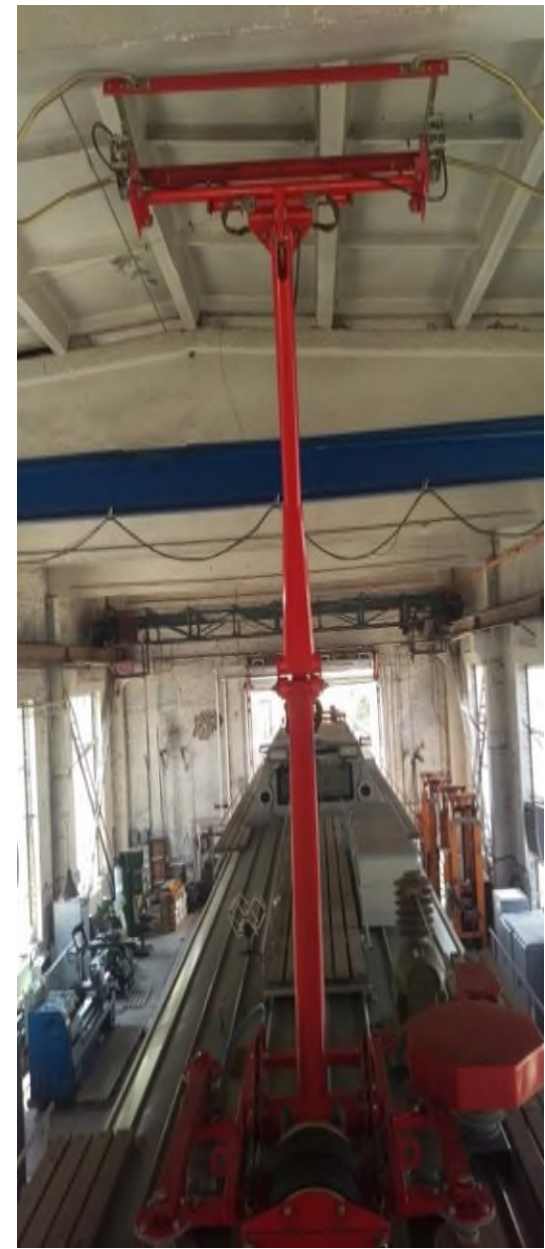

a

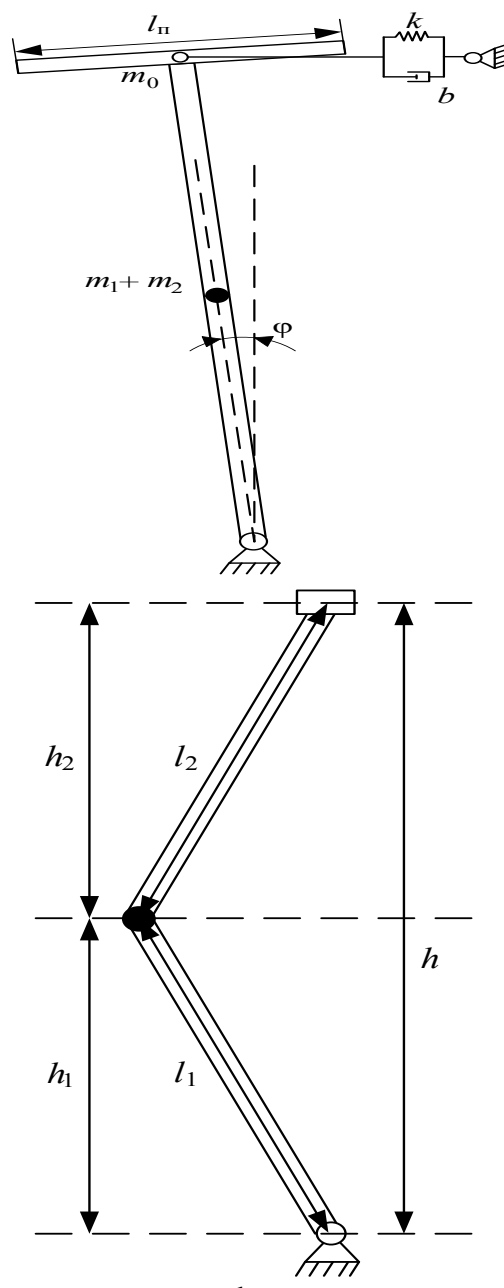

b

Fig. 3. Determination of transverse vibration indicators of a single - lever current collector: a current collector fixed at the maximum working height; b-replacement scheme for determining the moment of inertia of the current collector relative to the axis of the lower hinge.

Taking into account the accepted assumptions, the moment of inertia of the current collector in the transverse plane can be determined by the following expression:

$$
J_{m}=\frac{m_{1}}{12} \cdot h_{1}{ }^{2}+m_{1} \cdot\left(h_{1}+\frac{h_{2}}{2}\right)^{2}+\frac{m_{2}}{3} \cdot{h_{2}}^{2}+\frac{m_{0}}{12} \cdot l_{\Pi}^{2}+m_{0} \cdot\left(h_{1}+h_{2}\right)^{2}
$$

where $m_{1}-$ is the mass of the upper lever; $m_{2}$ - is the mass of the lower lever; $m_{0}$ - mass of the slide; $l_{\Pi}-$ length of the slide.

The solution obtained by using the substitutions: $\omega_{0}^{2}=\frac{\left(g \cdot\left(\left(m_{1}+m_{2}\right) \cdot h_{1}+m_{0} \cdot\left(h_{1}+h_{2}\right)\right)-k \cdot\left(h_{1}+h_{2}\right)^{2}\right)}{J_{m}}$ and $2 \cdot \beta=\frac{b \cdot\left(h_{1}+h_{2}\right)}{J_{m}}$ characteristic equation $\ddot{\varphi}+2 \cdot \beta \cdot \dot{\varphi}+\omega_{0}^{2} \cdot \varphi=0$ it showed that vibrations will be weakly damped. In this case, to calculate the rigidity, it is proposed to write an expression for determining the cyclic frequency of damped vibrations $\omega_{1}$ : 


$$
\omega_{1}=\sqrt{\omega_{0}^{2}-\beta^{2}}
$$

After substituting and converting an expression to the form:

$$
\begin{aligned}
k= & \frac{\sqrt{-J_{m}^{2}\left(h_{1}+h_{2}\right)^{4} \cdot\left(\left(\frac{b \cdot\left(h_{1}+h_{2}\right)}{2 \cdot J_{m}}\right)^{2}-\left(\frac{2 \pi}{T_{1}}\right)^{2}\right)}}{\left(h_{1}+h_{2}\right)^{4}}+ \\
& +\frac{g \cdot\left(h_{1}+h_{2}\right)^{2}\left(\left(m_{1}+m_{2}\right) \cdot h_{1}+m_{0} \cdot\left(h_{1}+h_{2}\right)\right)}{\left(h_{1}+h_{2}\right)^{4}}
\end{aligned}
$$

we can get numerical rigidity values by entering the data included in the expression.

The design parameters required for the calculation: $l_{1}-1.8 \mathrm{~m} ; l_{2}-1.95 \mathrm{~m} ; m_{1}-12 \mathrm{~kg}$; $m_{2}-12 \mathrm{~kg} ; m_{0}-17 \mathrm{~kg} ; l_{c}-2.0 \mathrm{~m}$, are accepted in accordance with the data of the current collector TA-160 - 2400. The remaining values included in the calculated expressions are determined on the basis of experimental studies [9], or using direct measurements (figure 4). Experimentally, the period of damped vibrations is determined using the expression:

$$
T_{10}=\frac{10}{T_{10}}
$$

where $T_{10}$ - the duration of ten vibrations determined by the registogram (figure $4 \mathrm{a}$ ).

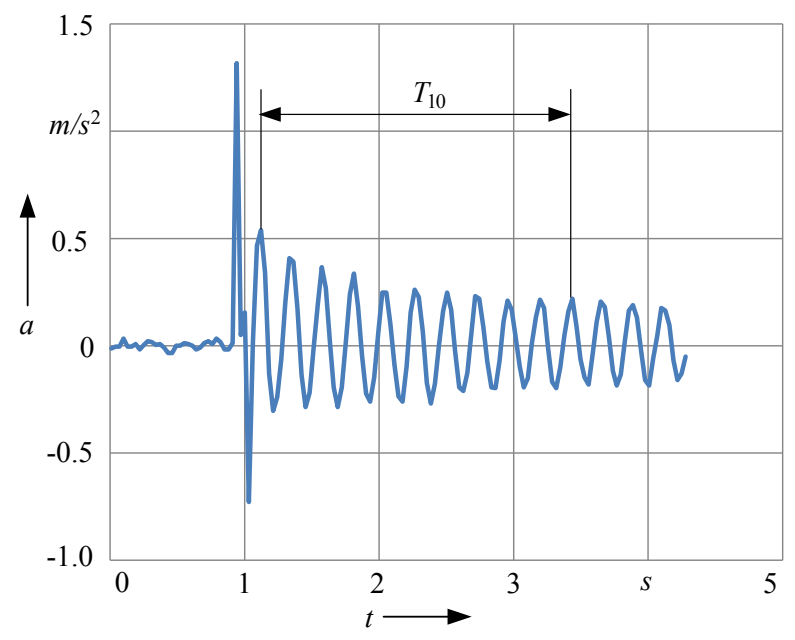

Fig. 4. Registration of transverse vibrations of the upper hinge of the current collector under the action of a single pulse.

Measurements carried out on a current collector with a single-lever design of the movable frame system showed that the circular frequency varies in the range from 29 to $42 \mathrm{rad} / \mathrm{s}$, depending on the height of the slider.

The rigidity determined by expression (4) in this case will be in the range from 17 to 24 $\mathrm{N} / \mathrm{mm}$, which meets the requirements of GOST 32204-2013 [3].

In accordance with the developed method, to determine the transverse rigidity of the system of movable frames of the current collector on the roof of an electric rolling stock, it is necessary to attach an accelerometer to the upper node [10]. Set the pressure in the pneumatic system so that the 
active static pressure will be in the range of $0 \pm 5 \mathrm{~N}$. Set the height position of the slide of current collector at the desired height. If necessary, we can fix the lower hinge of the movable frame system from turning around the axis using a clamp. After performing these procedures, it is necessary to run a program to determine the rigidity [11], and then, using a pulse action applied to the upper hinge, bring the current collector out of balance. After registering the transverse vibrations, the program recalculates the rigidity based on the current collector parameters previously entered in the database.

\section{Results}

Experimental studies conducted in the laboratory allow us to conclude that the lowest value of the transverse rigidity of the current collector corresponds to the maximum height position of the slide.

Thus, to check the rigidity of the current collector without removing from the electric rolling stock roof, it is sufficient to obtain the frequency of its transverse vibrations at the maximum working height, and then, using the given expressions, determine the numerical value. In case of negative value of the rigidity, the current collector must be dismantled with special equipment to identify the fault, and then eliminate the cause of the reduced rigidity.

Experimental studies performed in the laboratory "Contact networks and transmission lines" of Omsk State Transport University (OSTU), allowed comparing the rigidity of the system of moving frames of physical model of the current collector, measured in the traditional way, with those obtained according to the proposed method (figure 5).

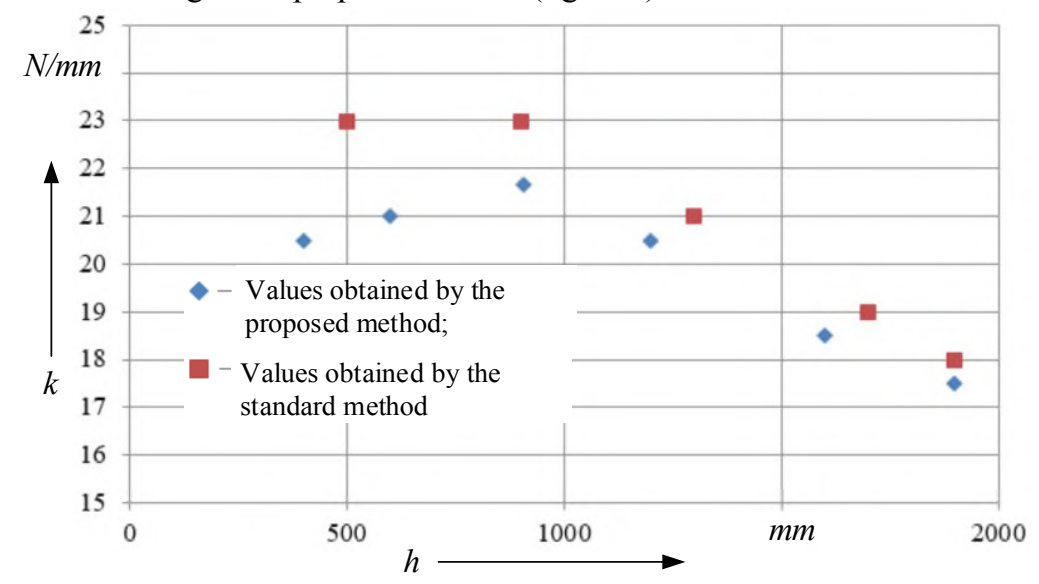

Fig. 5. Rigidity values of a single-lever current collector measured in the standard way and using the proposed method.

The results show a convergence sufficient for the application of the developed technique when checking the current collector rigidity after maintenance service (MS-2) or repair (TR-1; TR-2; TR-3). The practical value of the proposed method of rigidity control is to reduce the time spent on servicing current collectors, as well as to reduce the complexity of measuring the transverse rigidity of current collectors, a parameter that is one of the key parameters for mandatory confirmation of compliance according to GOST 32204-2013 "Current collectors of railway electric rolling stock".

Currently, the model of transverse vibrations of the current collector within the framework of the initiative research improved to ensure the applicability of the developed technique in the operation of current collectors of other structures, developed the device for registering vibrations of the current collector without removal from the electric locomotive. 


\section{Conclusion}

As a further direction of development of the topic it can be noted that the study of the spectral composition of vibrations, registered including on faulty current collectors, will highlight patterns corresponding to different faults, to make the classifier of conditions, to predict as data accumulates, their performance and thereby not only improve the productivity and efficiency of diagnostic procedures, but in accordance with the approved long-term development program of JSC "RZD" [12], go to the "digital railroad".

\section{References}

1. Decree of the RF Government dated 17.06.2008 No 877-R "Strategy of railway transport development in Russian Federation until 2030"

2. Decree of the President of the Russian Federation of 07.07.2011 No 899 (ed. of 16.12.2015) "On approval of priority directions for the development of science, technology and machinery in the Russian Federation and the list of critical technologies of the Russian Federation"

3. GOST 32204-2013 Current collectors of railway electric rolling stock. General technical conditions, 24, (2014)

4. A.N. Smerdin, A.S. Golubkov, V.M. Pavlov, I.E. Chertkov, A.V. Ryzhkov, Patent for utility model 178813 (RF), IPC G01M 17/08; B60L 3/12. Device for measure the rigidity of current collector, 2017125813; Appl. 18.07.2017; Publ. 19.04.2018. Bull. No 11

5. M.O. Kalashnikov, Diagnosis and quality control of building structures by vibrating method, 154 (2011)

6. V. I. Korobko, R. V. Aldushkin, O. V. Boyarkina ,Korobko, Experimental studies of steel trusses with parallel belts on static and dynamic effects, Izvestiya OrelSTU Series "Fundamental and applied problems of engineering and technology" 39-43, (2009)

7. A.N. Kuleshova, Longitudinal-transverse vibrations of a composite rod, 157, (2009)

8. A.G. Galkin, Theory and methods for calculating the processes of design and maintenance of the contact network, 370, (2002)

9. A. S. Golubkov, A. N. Smerdin, V. M. Pavlov, Improvement of test methods for current collectors of modern electric rolling stock, Electrification and development of power supply infrastructure for traction trains of high-speed and high-speed railway transport: thesis of report of VII Intern. Symposium "Eltrans-2013" 35-36, (2013)

10. O. A. Sidorov, V. M. Pavlov, A. N. Smerdin, A. S. Golubkov,Sidorov, Use of accelerometers for testing current collectors, Transport in Ural 46, 105-108 (2015)

11. A.N. Smerdin, A.S. Golubkov, A.V. Ryzhkov, Certificate about official registration of the computer program 2017661151 (RF). Software package for recording the longitudinal and transverse rigidity of a current collector - 2017616306. Appl. 30.06.2017; Publ. 04.10.2017

12. Order of the Government of the Russian Federation No 466-R dated 19.03.2019 "On approval of the development program of JSC "Russian Railways" until 2025 " 